\title{
Comparing Information Access Approaches
}

\author{
Matthew Chalmers \\ Department of Computing Science, University of Glasgow \\ matthew@dcs.gla.ac.uk
}

\begin{abstract}
Information retrieval, workflow, collaborative filtering and the path model can be considered as members of the family of approaches to information access. Although details of nomenclature and technique may vary, each is meant to provide people with access to useful information. In this paper we take a broad view over information access, drawing from philosophy and semiology in constructing a framework for comparative discussion. We use this framework to examine the information representations that underlie these four approaches, looking at phenomena included and excluded, the sharing of information amongst the community of use, interaction in terms of models of user activity and presentation of results, adaptation of system behaviour, and the inter-relationships of the representation's components. With deeper understanding of relative strengths and weaknesses, and characteristic emphases and assumptions, we can improve our selection, combination and development of information systems.
\end{abstract}

\section{INTRODUCTION}

Information access approaches such as information retrieval (IR), workflow, collaborative filtering (CF) and the path model have different notions such as 'relevant', 'timely', 'recommended', and so forth, but each has an underlying model of what information or meaning is involved in achieving essentially the same end: for a given person in a given context and with some 'content' or collection, make that person aware of some useful information. In this paper we try to take a wide and unifying view of how such approaches represent the entities involved in information access: the content, the people involved and the context of use. We see information science as part of a broader field dealing with symbols, interpretation and meaning. We wish to draw upon this wider field, taking particular account of the discourse in philosophy, semiology (or semiotics) and linguistics which have been developing for many centuries. More particularly we focus on the theory of the method or grounds of knowledge i.e. epistemology, and the theory of interpretation: hermeneutics. We wish to bridge between systems and use, and ultimately to enrich how we design and analyse information systems. We put forward a framework for discussing and analysing approaches to access and representation in information systems.

This work was influenced by another recent 50th, the anniversary of the publication of perhaps the most seminal document in information systems research: Vannevar Bush's As We May Think [Bush]. As We May Think is often held as foundational to hypermedia, the World Wide Web, and information retrieval. Interestingly, Bush does not put forward that the collection of links used in connecting hypermedia objects is a solution in itself. A 'sea of links' merely replicates the problem of having a sea of tuples or documents. Bush proposed that human activity in the form of 'trails' or 'paths' was the solution i.e. particular humanselected sequences of links associating information objects, and not the vast set of possible associations that hypermedia, databases and libraries provide. Note also that such a path does not directly involve content. It relies on human interpretation of content, but the meaning or value of an object is primarily given by the chosen context of a path. A link between two objects thus only has meaning within the context of a particular path of a particular person. The hypermedia and retrieval systems that declare an origin in Bush's work generally do not work with the phenomena of context and person as Bush proposed: context and person are not part of their underlying model of knowledge. Re-reading As We May Think and seeing this disparity between underlying models was one reason to look more closely at information access approaches' models of knowledge and interpretation.

We put the scope and dynamism of information representation at the centre of this paper's discussion. Firstly, how does the computer represent the people accessing and creating information? As was pointed out in [Winograd], the quality of this match, or mismatch, is a primary factor in determining the computer's ongoing interpretation of a user's information need, activity or interest. Are users represented as individuals, 
as stereotypes or in categories, or not represented at all? In other words, what phenomena are recorded as symbols to indicate the past and present information activity of each user? Secondly, what is included and excluded in each document or object, which indirectly may represent an aspect of the activity of one or more authors? Are the representations of content and users independent, and how do they originate and adapt? And, as various authors have discussed under the rubric of 'relevance,' how are these components combined to provide a person with useful information?

Complementary to discussion of how the computer represents user activity is the choice of how the system is presented to the user: what subsets and categories of the information representation should be the response of the system at each point in time? This can range from simply the name of a single object, to a little detail about a small set (e.g. an ordered list of document titles and keywords for the 'top ten' query matches), and on towards presenting the entire system e.g. a map of the collection, overlaid with patterns showing past and present user activity, and where one can zoom in on each object to read all contained detail.

Over the years, a number of reviews and discussions of relevance such as [Saracevic] and [Schamber] have shown the difficulty and complexity of defining relevance, and have warned against reliance on topical, impersonal and static notions of relevance. The continuing importance of these points is reinforced by recent empirical studies of users' relevance judgments such as [Wang], which demonstrated the importance of non-topical features such as recency, novelty, quality, availability, and authority. Wang also echoed earlier authors in saying that "IR systems must take users' cognitive and situational behaviours into consideration" and echoed Cooper's 1973 argument that "it is really documents with high utility, and not merely [topically] relevant documents, that the user wants to see" [Cooper].

It is remarkable that even though this kind of well researched and well argued criticism has been published for so long, it has not changed the mainstream practice of information science. In contrast to the obvious advances in computational power, our most commonly used information systems have changed little of their representational core during this time. Information retrieval systems have stayed close to the rather simple and static models of information representation that suited the capabilities of early computers. There are characteristic circumstances where they are still the most powerful and appropriate tools to use, but we are also aware that quite different tools have been developed to suit other uses and conditions. One goal of this paper is to explore the strengths, weaknesses and inter-dependencies of these tools and approaches. To achieve this goal, and to extend discussion of relevance, in the next section we draw from epistemology, semiology and hermeneutics in developing a tool or framework to examine the representations and assumptions that underlie information access approaches.

\section{A FRAMEWORK FOR DISCUSSION}

Hermeneutic theory is based on pragmatically accepting the complexity and subjectivity of human behaviour, of which informational activity is of course merely an instance. It focuses on behaviour as meaningful action, as acts of interpretation, and also emphasises subjectivity of interpretation. (We make particular reference here to the philosophical hermeneutics of Gadamer [Gadamer], to which [Warnke] is a fine introduction. [Grondin] covers hermeneutics more generally, and [Coyne] relates hermeneutics to the foundations of information science.)

Beyond content and topicality, use of information in a human activity involves a background of assumptions, abbreviations, the people involved, the other information that they share as part of their current activity, their organisational structure and practices, and so on, in endless detail. This view of the situated nature of activity is an antecedent of current HCI theory such as [Suchman]. Each user's interpretation involves their subjective understanding of the information's content, context and author, as well as understanding of other contexts of use of the information. The meaning of the information to a person is thus not an absolute, defined independently of other things and symbols, or as the unique and objective identifier for a 'thing in the world'. Instead, the meaning of a symbol or piece of information is inextricably dependent on the other symbols that make up the language and understanding of that person i.e. a system of relative differences and similarities between symbols define meaning in the way unique to each individual. By our actions and activities in the world, we display, test and adapt our understanding, but we can never 
prove our understanding to be absolutely true, or even to be identical in all detail to another's understanding. We can induce theories of what is true, but they are always potentially falsifiable.

One must also ask how such a relative system evolves and grows. The meaning of an individual symbol or text depends on the moment of interpretation and the understanding brought to it by the interpreter. But that understanding is itself the product of a history of interactions in language; interactions which themselves had to be understood in the light of earlier understanding. Furthermore, since the interactions in this circular process generally involve other people, learning from and adapting to them, we see the social aspect of meaning. This endless adaptive process of seeing the part in and through the whole is the hermeneutic circle. A consequence of this is that formal and objective abstractions, often seen as 'meta-information' that stands above the described phenomena, are pulled back down to the level of data again by human interpretation. When formal abstractions and meta-information are removed from controlled use, and people use them in their everyday work or activity, the hermeneutic circle brings those abstractions back down into the relative system of language. Formality is lost and metadata becomes data when control over interpretation passes from the system to the user. Since we are dealing with information systems that ultimately must pass information over to the human user, we therefore take a stance where there is no meaning for a symbol independent of human interpretation.

Hermeneutics stands in contrast to positivism that treats information as reducible to absolute, independent symbols. This stance maintains that if only the optimal analysis or abstraction could be made, the variability and subjectivity of use could be 'squeezed out' of the information representation. We would obtain an objective, predictive and formal model of information and its use. Hermeneutics sees this as excessively idealistic and reductionist, inadequately modelling the unavoidable complexity of language and behaviour. Hermeneutics takes a pragmatic stance, accepting that any fixed or formal representation of information brings with it both costs and benefits. Reduction from infinite detail to a finite representation involves the choice of which phenomena we will ignore, which we reduce by categorisation, and which we retain in detail because we assume such specificity is useful for our tasks. If we can use the same stereotype to represent each member of a category, on the assumption that each member is similar enough to the stereotype for our purposes, we make comparison and calculation more tractable. For example, finding documents that suit a given person's activity or need can be quickly done when we represent content and activity using the same small fixed vocabulary. If the similarity assumption is false, however, our system may efficiently describe and react to the sterotypical or 'average' situation, but no actual situation. Our system fits exactly with our model of the 'average person,' but not with anyone in particular.

We end this section with a list of landmarks or reference points that to some extent summarises the paper so far, but in following sections will be used to guide our analysis of four categories of information access approach:

- Phenomena: Which phenomena are represented or emphasised? Examples are the people involved, the context of work activity, the content of objects such as documents, and meta-information. How are phenomena interpreted: as abstract and objective, or as contextual and subjective with regard to each person involved?

- Sharing: Is the representation a shared resource that the entire community of users can draw from and add to, and hence use as a medium of communication?

- Interaction: Does each moment of use involve a summary or overview of the entire representation? Or do we consider only the most relevant details or subparts of the representation? If the latter, does this require explicit declaration and formalisation of relevance, or is this more passively determined e.g. by extrapolating from past activity?

- Adaptation: Is the representation of objects, people and their categories fixed a priori or does the system adapt with every use? How are objects added to or deleted from the representation?

- Relativity: Does the representation of each object depend on its differences and similarities with others? Before continuing, we briefly reflect on this list. No doubt incomplete, the list is offered as a rough map to initiate discussion and sharing of ideas, rather than as a script or formal checklist. Abstracting over and extending earlier sections, it is a rough prototype that can be expected to be adapted in the light of future discussion and experience. It also should not be taken as a strict partition of design concerns; clearly the listed points overlap with and depend on each other. 


\section{INFORMATION RETRIEVAL}

A stereotypical information retrieval system emphasises the content of documents, almost to the exclusion of other phenomena such as person and context, because topical relevance is in its foundations. Systems statistically smooth over or ignore what earlier users searched for and accessed, the identity and history of the particular person working now, the other tools and documents he or she is currently using, and so on. In early IR, each word or term in a collection had a single absolute representation, made independently of the user and his or her ongoing activity, and even of other terms. For example, "in practice the term-correlation problem is often solved, or circumvented, by assuming that the terms are in fact uncorrelated." [Salton:315] Documents were represented similarly, although such features as thesauri and weighting schemes involving cross-document statistics of term occurrence soon blurred the boundary between documents and between words, and created a more relativistic representation. The commonly used tf.idf weighting scheme is an example. A document is represented by a vector of pairs: each word or term occurring in it, and a weight for that term. The weight involves the term's frequency of occurrence within the document $(t f)$ multiplied by the inverse of its document frequency (idf), that describes what proportion of documents in the collection contain the term. Editing a document, adding a document to or deleting one from the collection implies adaptation in every other document, which makes the representation of information distinctly relativistic.

Interaction is driven by explicit queries in a formal language, which are generally represented identically to documents, as terms and weights-but only internally and temporarily. IR systems are meant to provide information of relevance to the user, yet the queries and query results, the primary representations of ongoing user activity, are not added to or used to adapt the collection. Some techniques, such as relevance feedback, reach back a very small way into the past e.g. back to the start of the current search session, but do not offer facilities for persistence, indexing and later retrieval. Filtering systems go some way further, changing the weights of the words in a person's filter with use. Also, some recent query expansion work reuses the past queries of others [Fitzpatrick]. Nevertheless, the author is aware of no systems that assimilate every user action and adapt the underlying document collection itself. In IR, support for the hermeneutic circle is thus weak.

Some work, taking account of cognitive and situational factors, has extended the range of phenomena representing documents and their use. In the Monstrat model for interface design [Belkin], functional modelling of the situation underlying activity was constrained by fact that information need may be very fluid and contextually defined — a result echoing the 'situated action' work of [Suchman]. The Mediator model of [Ingwersen92] tried to go further by formally modelling the reasons for and situation of the user's query or request. In Ingwersen's later Polyrepresentation model [Ingwersen94], the same information object can have multiple representations derived from object content as well as "from the cognitive space of a user." Ingwersen wishes to formally model the cognitive structures of people involved in the IR system as well as the system of categories and concepts that make up the system's model. His desire to formalise the reasons for and context of human behaviour represents a regression towards a positivistic stance. This is reflected in his view that cognitive structures can be transmitted from document author to system to reader, a notion that requires some absolute representation of cognitive structures which can be reliably packaged and transferred, which is quite at odds with the contemporary view of situated, subjective and relativistic knowledge.

The focus on content in categorising and defining the types of information e.g. image, audio and textual data, has led to category-specific and hence metrics of similarity specific to content type. Comparing objects that are thus considered to be of different types is made difficult e.g. the ASCII characters for the word "pipe" are not directly comparable with a picture of a pipe. Activity or need represented as an object of one type, e.g. a query as a term vector, is then difficult to use in retrieval of objects of different types e.g. image data. Many have tried to handle image data by directly analysing image content, but without overwhelming success. Others, as in the recent case of [Harmandas], use a blurring of the boundary of the document by analysing surrounding text e.g. content of textual pages within a distance of one or two Web links. Another approach has been to try to use metadata to formally and objectively describe information. For example, the PICS metadata format [Resnick97a], involves 'labels' (i.e. categories) to describe a web page or site e.g. suitability for children, whether it is commercial or not, and 'coolness'. Interestingly, Resnick and Miller suggest that there might be many rating services, each of which could choose its own rating vocabulary. When rating services are open to general use, metadata becomes just another aspect of a site's appearance 
i.e. data. Furthermore, even if the metadata describing a site is one thousandth the size of its referent, the vast size of the Web would lead to gigabytes of metadata. Ironically, we would then need 'metametadata' tools to find good rating services. We have not solved the problem, but deferred or even exacerbated it by adding to the indirection and complexity involved in acquiring utile information.

\section{WORKFLOW}

Workflow provides information access primarily by means of representations of organisational activity, based on a formalised categorisation of human speech and interaction. It stands out in its deliberate lack of flexible interpretation of human activity, affording control and uniformity of organisational behaviour, at the expense of personal improvisation and adaptation. Given the context of this paper, it also stands out in having philosophical hermeneutics explicitly mentioned as a motivating or foundational influence [Winograd].

In building the workflow model, Winograd and Flores used as foundations hermeneutics and the speech act theory of Austin and Searle. Austin related the interpretive actions discussed in hermeneutics to their consequent actions or commitments [Austin]. Searle then made a taxonomy based on recurrent patterns of speech acts [Searle]. Workflows are built by examining the interactions within an organisation and formalising the flow of information by means of these categories of recurrent interpretive actions. As was pointed out in [Suchman:41], however, Searle himself argued against the possibility of such formalisations of speech acts being used to construe the significance of any particular action or utterance. Winograd and Flores chose to ignore this, and de-emphasised the subjectivity of interpretation on the part of each user involved in an action or utterance. Even if such acts of interpretation can be categorised into simple groups, there is still the possibility that people will differ about which category an act belongs to. For example, an office worker may send a report on a paper jam in a printer to a technical support group. The sender may see the report as a demand for immediate action but the receiver, busy with repairing a disc crash, may treat it merely as a suggestion that perhaps at some future date a better printer might be made available.

One can see workflow as focusing predominantly on one aspect of context, as one can consider a person's formal organisational role as a contextual feature. Each such organisational role has a finite set of states and state transitions, and some states are associated with an ability to accept a new item of work. Information is passed to a person because of his or her declaration of being in an accepting state, and work is waiting 'upstream' in the model. Interaction involves presentation of information to a member of the organisation because, according to the structure and current state of workflow model, it is the most timely and thus utile object. The effectiveness of modelling depends on how well the static model of roles and states fits individuals and their work. [Button] describes a study of a workflow system in use at a large printing plant where the insufficiently flexible 'standard operating procedures' for print jobs were often circumvented to handle contingencies such as printer breakdowns and fluctuations in job arrival rates and resource requirements. For example, in order to get the real work done, printer operators represented fake people in the system (with names such as 'Mickey Mouse') so as to do 'illegal' dynamic job rescheduling.

A workflow combines user activity and the information involved in it, although overview and explicit modification of that representation are not generally offered to users. Also, there is no inherent dynamism in the workflow model that would let it automatically adapt with use. The interpretation of people's roles and information flow is designed, then fixed. Again we see a static formalisation made a priori and for all people involved. One can thus say that the hermeneutic circle is weak or broken.

Recent work has tried to reduce this weakness, for example in the Freeflow system [Dourish] where the representation of activity puts weaker constraints on actual activity than in most workflow systems. Users can choose in what order and overlap of tasks best suits their current situation, as long as they do not break the requirements for consistency and correctness in interactions with others. Other work has focused on making the representation itself more flexible, for example in the 'generalised process structure grammars' of [Glance]. Formal graphs and grammars still represent activity, but since users may individually customise and edit these representations of work - assuming they can handle the tools of customisationthen these formalisations are taken out of the hermeneutic circle of the organisation. They are no longer shared across the organisation and part of the common language of everyday activity. Metadata becomes data again, and organisational consistency and control are lost. As these grammars proliferate, another 
metametadata system may then be needed to support the storage, retrieval and communication of the personal rules of many people, as the workflow system is not a common information space where shared experience aids the task of designing work patterns.

\section{COLLABORATIVE FILTERING}

Collaborative filtering (CF) is a burgeoning information access approach based on patterns of subjective ratings of information objects. Its power and significance seem somewhat undervalued inside the mainstream IR community, although CF shows a powerful holism with regard to types of data such as books, films and music. Relying on identifiers and ratings affords metrics of similarity for data of heterogeneous content. A textual document about pipes can be associated with a picture of a pipe if they are used or rated similarly. Perhaps the earliest published collaborative filtering system was the Tapestry system for accessing e-mail and bulletin board messages [Goldberg]. Some of the currently best known research systems are described in [Resnick97b], and another widely known system is the book recommender at Amazon.com, a web-based bookshop.

In a typical CF system, recommendations are specific to the person involved but independent of their particular current activity or context. Interaction is driven by building up a profile of ratings. Formalising why one liked or disliked an object is avoided or reduced, for example by selecting from a five point rating scale from 'very good' to 'very bad,' and so one can react according to one's informal understanding. Representation with a rating scale is still categorisation, and is still prone to subjective variation e.g. some people will tend to use extreme rating values, while others will be more comfortable staying in the middle of the range. Nevertheless, powerful schemes of interpretation can be built upon representations such as one profile per person, and a binary rating scale passively obtained with each of a well-defined set of actions. An example is Amazon.com's book recommender where each book purchase is treated as an interpretive act (i.e. as giving it a good rating) that adds to the purchaser's profile.

Similarity of two profiles is considered high if they have similar ratings for the same objects. Given a target profile, a set of 'neighbours' (of some fixed and usually small maximum size) is then determined, being the most similar profiles. This is sometimes described as a way of finding similar people-indeed, the first collaborative filtering system is said to have been an 'automatic dating agency.' Ordinarily, however, the system presents to a user a small number of objects that similar people rated highly but that he or she has not yet rated. Sometimes one can explicitly choose the subset of people from whom profiles should be drawn, allowing use of one's knowledge of colleagues' and friends' expertise.

Rating a recommendation feeds back into the process of adaptation of one's profile, neighbour set, neighbours' neighbour sets and beyond. Ripples of change spread out, demonstrating adaptation and relativity. The hermeneutic circle is strong here and, again showing an affinity with hermeneutics, there is no meaning for an object independent of at least one person's interpretation. Note a consequence: an object with no ratings cannot be recommended. Initial ratings must be generated manually, through passive rating of actions in another information access system (e.g. book purchases, above) or other means external to the CF system.

Sets of ratings are useful objects in themselves, and system use involves a kind of sharing, but generally they are not put in common for view, discussion and more direct manipulation. Occasionally, however, we can treat a rated object as well as each of its contained objects as symbols, holistically. Many people, for example, maintain a web page of 'hot links' or bookmarks. If this page instead displayed the user's profile in a CF system, and the URL for this page was amongst the symbols manipulated within the system, then we would see metadata slipping down to being data again. Accessing such a page might suggest a passive rating of the pages it references.

Selection of the people involved in recommendation may afford some 'steering', but explicit control over system behaviour is more often constrained to rating objects so as to adapt one's profile. This may be difficult as CF generally treats all one's ratings equally, although one might assume that more recent ratings or activity should be given greater weight. Taking more account of the temporal order of activity is at the core of the path model, discussed in the next section. 


\section{THE PATH MODEL}

The path model is a recently developed approach to information access that has its origins in collaborative filtering. It shows an emphasis on subjectivity, relativity and adaptation in information representation. A prototype system for logging individuals' explicit access of URLs, recommending URLs based on the context of an individuals' recent activity, and visualising logs was described in [Chalmers]. This system was limited to context and person. A 'production' version to be used by a group of roughly 300 people is now being developed, and research prototypes continue to evolve. In this section we will give some details of our most recent research prototype, such as its extension to handle content, but since this paper focuses on general approaches, we leave fuller details to a forthcoming publication.

The use of 'path' in the name of this approach refers partially to As We May Think, where information objects were linked together into paths or trails. Context and person were at the core of the underlying model of knowledge, rather than content. The second reference is based in the model's origin. It was developed as an analogy of a theory of urban structure, use and development: the 'space syntax' of [Hillier]. Space syntax puts people's movement at the centre of a theory of city structure and development, and uses people's paths through the city as expressions of their activities, interests, and associations. Hillier deliberately avoids a priori categorisation of paths, instead relying on accumulating statistics of movement and activity by members of the public. Analysis is in terms of the configuration of buildings and streets rather than the content or functionality of individual urban elements. He emphasises the importance of considering the extended paths individuals take rather than transitions between pairs of city elements. The analogy with information representation relies on treating histories of information use like paths through the city, treating information objects like spatial forms, and language as the city.

Like CF, the path model primarily interprets information by means of the people who use it. A path is a time-ordered history of a person's use of information objects. The use of symbols such as words, URLs and filenames displayed in a Web browser and the xemacs editor are logged over time. The log serves as the representation of user activity. Each path entry is associated with the system's observation of a user accessing a URL in a web browser, or switching between editor buffers. Logging of content assumes that all that is displayed is seen: a weak assumption for long documents until we can track where the user scrolls and jumps. By default, each path is visible to all those who contribute paths i.e. the set of paths is treated a shared resource. Privacy is therefore an issue. Each user can turn path logging on and off at will, and we are developing tools to make it easier for a user to edit his or her path.

Unlike CF, context or current activity is an essential part of the approach. Each instance of a symbol's use is associated with that person's temporally closest path entries. We then use patterns of symbol recurrence in determining relevance and information need, by treating the most recent sequence of path entries as an implicit request for recommendations. A slider in the recommender's interface allows user selection of the time period that delimits 'recent' symbols. The system periodically takes the recent symbols and searches for past occurrences of each one. This search can cover one's own path and/or a selection of others'. We are currently developing tools for explicitly adding to and deleting from the set of recent symbols i.e. making the expression of information need as explicit or as passive as required.

The system collects the context of each past occurrence of each recent symbol i.e. each past occurrence's 'window' of path entries that subsequently occurred within a chosen number of minutes, as set using another slider. The system tallies the symbols collected from these windows, discards any symbols that were recently used-in effect, recent symbols form an adaptive stop list—and then the system presents the remaining symbols as a ranked recommendation list. We also optionally present $2 \mathrm{D}$ visualisations of the windows around recommended symbols. After display, the system sleeps for a time then wakes, collects a new set of recent symbols and begins again. Another slider sets the sleep time.

Echoing Cooper's words quoted in the Introduction, we focus not on topical relevance, but on utility. This is because when past occurrences are near to each other, their windows overlap. Symbols within overlaps get higher tallies. Highly ranked symbols tend to be, therefore, those symbols most consistently used in the contexts most similar to recent activity. We assume that consistency of use in similar contexts is the best indicator of appropriate (literally, utile) recommendations. 
While recurrence statistics form categorisations or formalisations, statistics over path entries and their patterns are not gathered a priori but anew for each person at the time of, and using the context of, each recommendation operation. As with collaborative filtering, each new extension to a path changes the pattern of symbol co-occurrences throughout the shared set of paths. Even if you have not accessed new information recently, your recommendations may still vary as other people's activities change the path configuration. Thus a path system adapts with every use, strengthening the hermeneutic circle. The 'meaning' of a symbol is determined by its pattern of occurrences, and hence its pattern of co-occurrence with other symbols. Symbols are thus represented relatively, not absolutely. There is no meaning for a symbol independent of paths and, consequently, of individuals' interpretation and use.

As with profiles in collaborative filtering, we should consider the 'metadata' of paths as objects within the path model. Recording the choice of whose paths one uses, and whose recommendations one uses, should be logged in one's path. Rather than fight or ignore it, we can then make good use of path meta-information slipping down to be information, recommending the names of people whose expertise might be utile in the current context.

Also like CF, the path model is dependent on external systems to initialise and extend the set of symbols recorded and used in recommendations. Paths and CF would be useless without people's use of Web search engines and browsers, and indeed mail tools, magazines, journals and so forth. We see this not as a weakness, but as realistic and pragmatic acceptance of the interdependence and holism of tools, and their embedding in the wider world. We interleave information tools in everyday use, and the sets of objects they operate on overlap. We should work with this interdependence, not against it.

\section{CONCLUSION}

We have discussed similarities and differences in 'family characteristics' shared by four information access approaches. By having a framework to discuss their strengths and weaknesses we can better understand their interdependencies and potential combinations. Most obviously, approaches based on relativistic information representations such as collaborative filtering and path model depend on other approaches and systems for initialisation and extension. Conversely, approaches that abstract over person and context, such as IR, can be applied when no human interpretation or use has yet been recorded. Since workflow, CF and the path model rely less on objective content, they can more easily handle heterogeneous data. Approaches such as workflow, which strongly formalises contextual and personal detail, and traditional IR, which deemphasises or ignores it, come into play when utility is more objective.

The subjectivity and adaptation of $\mathrm{CF}$ and the path model link them to hermeneutics more than positivism and its tendency to rely on representations that are objective and fixed. CF and paths rely on features discarded in IR and workflow, and vice versa. Information access approaches are interdependent tools to be used singly or in combination as appropriate. Understanding their characteristics, strengths and weaknesses should also help in the generation of new members of the family of information access approaches.

By looking at information access from a wider perspective, we have tried to increase or reinforce the reader's awareness of the utility of philosophy and semiology in information science. We wish to add them to the theories and techniques that are ready to hand in our field, and collectively treat them as interdependent intellectual tools to be used singly or in combination according to their strengths and weaknesses. In this way we can strengthen the connections between information systems and their use, between the theory and practice of information science, and between information science and the broader field dealing with symbols, interpretation and meaning.

\section{REFERENCES}

[Austin] Austin, J.L. How to Do Things with Words, Harvard U. Press, 1962.

[Belkin] Belkin, N., Brooks, H., and Daniels, P. Knowledge Elicitation Using Discourse Analysis, Intl. J. Man-Machine Studies, 27:127-144, 1987.

[Bush] Bush, V. As We May Think, Atlantic Monthly, July 1945. Reprinted in ACM Interactions 3(2), March 1996, 37-46. 
[Button] Bowers, J., Button, G., \& Sharrock, W. Workflow from Within and Without: Technology and Cooperative Work on the Print Industry Shopfloor, Proc. 4th Euro. Conf. On Computer Supported Collaborative Work (ECSCW95), 51-66, 1995.

[Cooper] Cooper, W. On Selecting a Measure of Retrieval Effectiveness. J. ASIS, 24:87-100, 1973.

[Chalmers] Chalmers, M., Rodden, K. \& Brodbeck, D. The Order of Things: Activity-Centred Information Access, Proc. 7th Intl. World Wide Web Conference (WWW7), 1998, 359367.

[Coyne] Coyne, R. Designing Information Technology in the Postmodern Age, MIT Press, 1995.

[Fitzpatrick] Fitzpatrick, L. \& Dent, M. Automatic Feedback using Past Queries: Social Searching?, Proc. ACM SIGIR 97, 306-313, 1997.

[Gadamer] Gadamer, H.-G., Truth and Method, 2nd edn., trans. J. Weinsheimer \& D. Marshall, Crossroad, 1989.

[Goldberg] Goldberg, D., et al. Using Collaborative Filtering to Weave an Information Tapestry, Comm. ACM 35(12), December 1992, 61-70.

[Grondin] Grondin, J. Introduction to Philosophical Hermeneutics, trans. J. Weinsheimer, Yale U. Press, 1994.

[Harmandas] Harmandas, V., Sanderson, M., and Dunlop, M. Image Retrieval by Hypertext Links, Proc. ACM SIGIR 97, 296-303, 1997.

[Ingwersen92] Ingwersen, P. Information Retrieval Interaction. Taylor Graham, London, 1992.

[Ingwersen94] Ingwersen, P. Polyrepresentation of Information Needs and Semantic Entities: Elements of a Cognitive Theory for Information Retrieval Interaction, Proc. ACM SIGIR 94, 101110, 1994.

[Resnick97a] Resnick, P. \& Miller, J. PICS: Internet Access Controls Without Censorship, Comm. ACM 39(10), October 1996, 87-93.

[Resnick97b] Resnick, P. \& Varian, H. (eds.) Special Issue of Comm. ACM on Recommender Systems, 40(3), March 1997.

[Salton] Salton, G. Automatic Text Processing, Addison Wesley, 1989.

[Saracevic] Saracevic, T. The Concept of "Relevance" in Information Science: A Historical Review. In: Introduction to Information Science, 111-151, Bowker, New York, 1970.

[Schamber] Schamber, L., Eisenberg, M. \& Nilan, M. A. Re-examination of Relevance: Towards a Dynamic, Situational Definition. Information Processing \& Management, 26:755-776, 1990.

[Searle] Searle, J. A Taxonomy of Illocutionary Acts, in K. Gunderson (ed.), Language, Mind and Knowledge, U. Minnesota, 1975, 344-369.

[Wang] Wang, P. The Design of Document Retrieval Systems for Academic Users: Implications of Studies on Users' Relevance Criteria, Proc. ASIS 97, 162-173, 1997.

[Winograd] Winograd, T. \& Flores, F. Understanding Computers and Cognition, Addison Wesley, 1986. 